\title{
Global risk factor rankings: the importance of age-based health loss inequities caused by alcohol and other risk factors
}

\author{
Kevin D Shield ${ }^{1,2,3^{*}}$ and Jürgen Rehm ${ }^{1,2,3,4,5,6}$
}

\begin{abstract}
Background: Achieving health equity is a priority of the World Health Organization; however, there is a scant amount of literature on this topic. As the underlying influences that determine health loss caused by risk factors are agedependent, the aim of this paper is to examine how the risk factor rankings for health loss differ by age.

Methods: Rankings were based on data obtained from the 2010 Global Burden of Disease study. Health loss (as measured by Disability Adjusted Life Years lost) by risk factor was estimated using Population-Attributable Fractions, years of life lost due to premature mortality, and years lived with disability, which were calculated for 187 countries, 20 age groups and both sexes. Uncertainties of the risk factor rankings were estimated using 1,000 simulations taken from posterior distributions

Results: The top risk factors by age were: household air pollution for neonates 0-6 days of age [95\% uncertainty interval (UI): 1 to 1]; suboptimal breast feeding for children 7-27 days of age (95\% UI: 1-1); childhood underweight for children 28 days to less than 1 year of age and 1-4 years of age (95\% UI: $1-2$ and 1-1, respectively); iron deficiency for children and youth 5-14 years of age (95\% UI: 1-1); alcohol use for people 15-49 years of age (95\% UI: 1-2); and dietary risks for people 50 years of age and older (95\% UI: 1-1). Rankings of risk factors varied by sex among the older age groups. Alcohol and smoking were the most important risk factors among men 15 years of age and older, and high body mass and intimate partner violence were some of the most important risk factors among women 15 years of age and older.

Conclusions: Our analyses confirm that the relative importance of risk factors is age-dependent. Therefore, preventing harms caused by various modifiable risk factors using interventions that target people of different ages should be a priority, especially since easily implemented and cost-effective public health interventions exist.
\end{abstract}

Keywords: Health equity, Global Burden of Disease, Disability Adjusted Life Years, Risk factor, Alcohol

\section{Background}

Achieving health equity is a top priority of the World Health Organization (WHO), as achieving health targets without an equitable distribution is of limited value [1]; however, there is a scant amount of literature on global health equity. Accordingly, the WHO has prioritized identifying and quantifying the determinants of health inequities [1,2], and, thus, an investigation

\footnotetext{
*Correspondence: kevin.shield@mail.utoronto.ca

${ }^{3} \mathrm{PAHO} / \mathrm{WHO}$ Collaborating Centre for Mental Health and Addiction,

33 Russell Street, Toronto, ON M5S 2S1, Canada

Full list of author information is available at the end of the article
}

of the distribution of health loss caused by risk factors among sub-populations can increase our understanding of how health inequities arise and how best they should be addressed [3-5]. The 2010 Comparative Risk Assessment (CRA) publication ranked risk factors by health loss [measured as Disability Adjusted Years of Life (DALYs) lost] [6]; however, as the underlying influences that determine health loss caused by risk factors are age-dependent (namely, exposure, and individual vulnerability to, and health outcomes from, causally-related diseases and injuries), the rankings of risk factors are hypothesized to depend on age. Accordingly, we examined the rankings of 
risk factors based on the DALYs lost by age based on the 2010 CRA findings.

\section{Methods}

Data were obtained from the 2010 Global Burden of Disease (GBD) study, for which the authors of this article played a role in the estimation of the burden of disease attributable to alcohol consumption (see [7] for a general overview of the methods used in the 2010 GBD study). The 2010 GBD CRA study estimated the number of DALYs lost by risk factor by calculating the number of years of life lost (YLL) due to premature mortality (see [8, 9] for more information on mortality data collection and estimation) and the number of years lived with disability (YLD) (see $[10,11]$ for more information on morbidity data collection and estimation), and also calculated the mortality and morbidity Population-Attributable Fractions (PAFs) by risk factor for 187 countries, 20 age groups and both sexes. PAFs represent the burden of a disease (either mortality or morbidity) that would not be present under a counterfactual scenario of everyone being exposed to the theoretical-minimum-risk exposure distribution (the amount of exposure that leads to the lowest burden of disease). For alcohol and tobacco in particular, this theoretical-minimum-risk exposure distribution assumed that every person abstained from alcohol and tobacco for their entire life, while for risk factors such as diet and blood pressure, the theoretical-minimum-risk exposure distribution assumed the healthiest blood pressure and dietary intake. For each risk factor, PAFs for mortality and morbidity were calculated using prevalence of exposure status and exposure distributions for people who were exposed to a risk factor (see [6] for information on data sources of the exposure status) and using the relative risks (RR) for each disease condition and injury that was deemed to be causally related to the risk factor (see [6] for a list of diseases, conditions and injuries causally related to each of the risk factors examined). The mortality and morbidity risk factor PAFs were then applied to the number of YLL and YLD respectively, with the resulting YLL and YLD being summed to estimate the DALYs lost attributable to each risk factor.

To estimate the uncertainty of the risk factor rankings, simulation analyses were employed using 1,000 draws from the posterior distribution of exposure, RRs, YLL and YLD for each age, sex and country. Simulations accounted for the correlation of uncertainty between measures. The 2.5 and 97.5 percentiles of the simulations were used as an indicator of the $95 \%$ uncertainty intervals (UI).

\section{Ethics}

As this was an analysis of secondary data, no ethics approval from the Centre for Addiction and
Mental Health (the authors' main research institution) was required.

\section{Results}

The top risk factors by age were: insufficient weight for children 5 years of age and younger (95\% UI: 1-1); iron deficiency for children and youth 5-14 years of age (95\% UI: $1-1)$; alcohol use for people $15-49$ years of age (95\% UI: $1-2$ ); and dietary risks for people 50 years of age and older (95\% UI: 1-2). In addition, smoking and high blood pressure had a greater impact on people in older age groups. As indicated above, alcohol (the fifth leading cause of health loss (95\% UI: 4-7)) had the greatest effect on people in young adulthood up to 49 years of age. Figure 1 outlines the risk factor rankings by age in 2010 .

Rankings of risk factors varied by sex for older age groups only. The risk factor rankings for children and youth (14 years of age and younger) showed little variation by sex. Among older age groups, alcohol, smoking and occupational risks were more important risk factors among men 15 years of age and older and high body mass index and intimate partner violence were more important risk factors among women 15 years of age and older. See Additional file 1: Figure A1 and Additional file 2: Figure A2 for the risk factor rankings in 2010 by age for women and men respectively.

\section{Discussion}

Our analysis confirms that the relative importance of risk factors is age-dependent. The findings concerning the top five risk factors, namely dietary risks, high blood pressure, smoking, household air pollution, and alcohol are especially important as a large variance in the risk factor rankings by age was observed. These differences in risk factor rankings by age should be taken into account, especially as utilitarian ageism is often observed in medical practice which leads to the prioritization of treatments for health loss among the young (as the old have lived longer) $[12,13]$.

Furthermore, the risk factor rankings for children and youth (14 years of age and younger) showed little variation by gender, while the risk factors for people 15 years of age and older showed a large variation by gender. This is likely due to risk exposure not varying by gender for children and youth (14 years of age and younger) when compared to the risk exposure differences by gender for people 15 years of age and older [14]. In particular, smoking and alcohol-two risk factors more prevalent among men than among women 15 years of age and older [15, 16] - caused a relatively larger burden of disease when compared to other risk factors for men when compared to women. Intimate partner violence was a more important risk factor among women 15 years of age and older, as women are much more likely to be the target of 


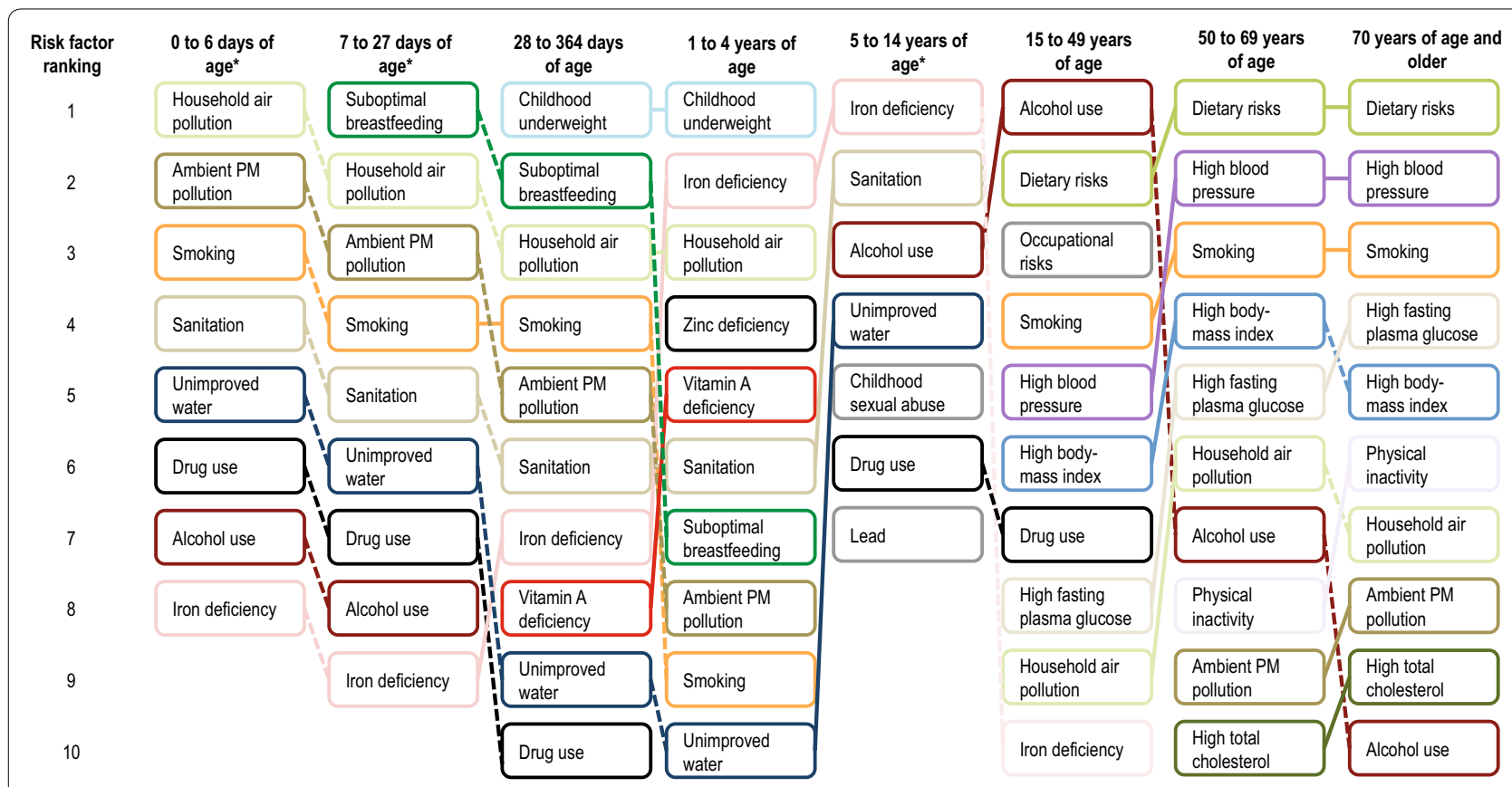

Figure 1 Global risk factor rankings (top 10) for the total burden of disease (measured in Disability Adjusted Life Years lost) by age in 2010. Asterisks data for exposure and risk relationships for all risk factors are not available for these groups, and thus fewer than ten risk factors are presented

intimate partner violence [17]. Lastly, high body mass index affected women more than other risk factors when compared to men; other risk factors such as smoking and alcohol caused a smaller relative burden of disease among women.

It should be noted that there were some limitations respecting the estimation of the burden attributable to alcohol consumption in the 2010 GBD study. Specifically, the burden attributable to alcohol consumption is likely underestimated, as conditions such as alcohol abuse and HIV/AIDS were not included in these calculations, and neither were any mental health conditions other than alcohol dependence (for a more recent estimate including these conditions see [18]). Furthermore, with respect to risk factors for neonates 0-6 days of age, children 6-27 days of age and children and youth 5-14 years of age, data were missing on the exposure to certain risk factors and risk relationships, and, therefore, calculation of the effects of these risk factors was impossible. For instance, the effects of smoking on children, youth and young adults aged 5-25 years was set to zero, even though tobacco smoking is likely to cause some burden of disease among people in this age group.

Health inequities should also be considered in light of the feasibility and effectiveness of interventions. As recommended by the WHO, effective interventions are replicable, sustainable, scalable, and politically, economically and technically feasible [1]. Of the top risk factors, alcohol and smoking interventions rank high on these dimensions. Lastly, several alcohol and smoking interventions were designated as "best buys" by the WHO as they are more cost-effective than most other interventions designed for other risk factors [19]; even the more cost-effective interventions for dietary risks, high blood pressure, and household air pollution are costly when compared to the most cost-effective interventions for alcohol and tobacco.

Furthermore, public health policy measures aimed at smoking and alcohol, such as advertising restrictions, are more prevalent for smoking than similar public health policy measures aimed at alcohol consumption. (This may be due, in part, to alcohol having a protective effect on ischemic cardiovascular diseases and diabetes at low consumption amounts [20]). Additionally, the fact that alcohol use is the top risk factor among people 15-49 years of age is important, as investment in preventing mortality from injury has fallen behind other causes of death, such as HIV/AIDS and reproductive health [21], and mental health concerns have been overlooked in terms of public health programming, especially in young people [22] where injuries and neuropsychiatric conditions are greatly impacted by alcohol consumption [23].

\section{Conclusion}

Although the risk factor rankings by age are important for equity considerations, analyses are needed to 
determine the underlying causes of health loss by dimensions other than age, such as by income, education, and race/ethnicity [24].

\section{Additional files}

Additional file 1: Figure A1. Global risk factor rankings (top 10) for the total burden of disease (measured in Disability Adjusted Life Years lost) by age for women in 2010.

Additional file 2: Figure A2. Global risk factor rankings (top 10) for the total burden of disease (measured in Disability Adjusted Life Years lost) by age for men in 2010.

\section{Abbreviations}

CRA: Comparative Risk Assessment; DALYs: Disability Adjusted Years of Life; GBD: Global Burden of Disease; PAFs: Population-Attributable Fractions; RR: relative risks; UI: uncertainty intervals; WHO: World Health Organization; YLD: years lived with disability; YLL: years of life lost

\section{Authors' contributions}

KS and JR conceptualized the overall article, acquired all data, and contributed to the methodology. KS performed all analyses. KS and JR contributed to the writing of the manuscript and approved the final version. Both authors read and approved the final manuscript.

\section{Author details}

${ }^{1}$ Centre for Addiction and Mental Health (CAMH), Toronto, Canada. ${ }^{2}$ Institute of Medical Science, University of Toronto, Toronto, Canada. ${ }^{3} \mathrm{PAHO} / \mathrm{WHO}$ Collaborating Centre for Mental Health and Addiction, 33 Russell Street, Toronto, ON M5S 2S1, Canada. ${ }^{4}$ Dalla Lana School of Public Health (DLSPH), University of Toronto, Toronto, Canada. ${ }^{5}$ Institute of Clinical Psychology and Psychotherapy, Technische Universität, Dresden, Germany. ${ }^{6}$ Department of Psychiatry, University of Toronto, Toronto, Canada.

\section{Acknowledgements}

The authors thank the numerous individuals who worked on the 2010 Global Burden of Disease study, especially Drs. Christopher Murray, Theo Vos, Stephen Lim, Haidong Wang, Alan Lopez, Majid Ezzati and Mohsen Naghavi.

\section{Compliance with ethical guidelines}

\section{Competing interests}

The authors declare that they have no competing interests.

Received: 14 June 2014 Accepted: 7 May 2015

Published online: 09 June 2015

\section{References}

1. World Health Organization (2010) Equity, social determinants and public health programmes. World Health Organization, Geneva

2. World health Organization (2008) Closing the gap in a generation: health equity through action on the social determinants of health. Commission on Social Determinants of Health Final Report. World Health Organization, Geneva

3. Gore FM, Bloem PJ, Patton GC, Ferguson J, Joseph V, Coffey C et al (2011) Global burden of disease in young people aged 10-24 years: a systematic analysis. Lancet 377(9783):2093-2102
4. Murray CJ, Gakidou EE, Frenk J (1999) Health inequalities and social group differences: what should we measure? Bull World Health Org 77(7):537-543

5. Asada Y (2005) A framework for measuring health inequity. J Epidemiol Community Health 59(8):700-705

6. Lim SS, Vos T, Flaxman AD, Danaei G, Shibuya K, Adair-Rohani H et al (2012) A comparative risk assessment of burden of disease and injury attributable to 67 risk factors and risk factor clusters in 21 regions, 1990-2010: a systematic analysis for the Global Burden of Disease Study 2010. Lancet 380:2224-2260

7. Murray CJ, Ezzati M, Flaxman AD, Lim S, Lozano R, Michaud C et al (2012) GBD 2010: design, definitions, and metrics. Lancet 380(9859):2063-2066

8. Lozano R, Naghavi M, Foreman K, Lim S, Shibuya K, Aboyans V et al (2012) Global and regional mortality from 235 causes of death for 20 age groups in 1990 and 2010: a systematic analysis for the Global Burden of Disease Study 2010. Lancet 380(9859):2095-2128

9. Wang $H$, Dwyer-Lindgren L, Lofgren KT, Rajaratnam JK, Marcus JR, LevinRector A et al (2013) Age-specific and sex-specific mortality in 187 countries, 1970-2010: a systematic analysis for the Global Burden of Disease Study 2010. Lancet 380(9859):2071-2097

10. Vos T, Flaxman AD, Naghavi M, Lozano R, Michaud C, Ezzati M et al (2012) Years lived with disability (YLDs) for 1160 sequelae of 289 diseases and injuries 1990-2010: a systematic analysis for the Global Burden of Disease Study 2010. Lancet 380(9859):2163-2196

11. Murray CJL, Vos T, Lozano R, Naghavi M, Flaxman AD, Michaud C et al (2012) Disability-adjusted life years (DALYs) for 291 diseases and injuries in 21 regions, 1990-2010: a systematic analysis for the Global Burden of Disease Study 2010. Lancet 380:2197-2223

12. Harris J (1985) The value of life: an introduction to medical ethics. Routledge, London

13. Tsuchiya A (2000) QALYs and ageism: philosophical theories and age weighting. Health Econ 9(1):57-68

14. Lopez AD, Mathers CD, Ezzati M, Jamison DT, Murray CJL (2006) Global and regional burden of disease and risk factors, 2001: systematic analysis of population health data. Lancet 367(9524):1747-1757

15. World Health Organization (2014) Global status report on alcohol and health. World Health Organization, Geneva

16. World Health Organization (2015) Global health observatory (GHO) data: prevalence of tobacco use. World Health Organization, Geneva

17. Hattery A (2009) Intimate partner violence. Rowman \& Littlefield, Maryland

18. Rehm J, Shield KD, Stevens G (2014) Health consequences. In: World Health Organization (ed) Global status report on alcohol and health. World Health Organization, Geneva, pp 45-57

19. World Health Organization: Global status report on noncommunicable diseases (2010) Description of the global burden of NCDs, their risk factors and determinants. World Health Organization, Geneva, p 2011

20. Roerecke M, Rehm J (2012) Alcohol intake revisited: risks and benefits. Curr Atheroscler Reports 14(6):556-562

21. Patton GC, Coffey C, Sawyer SM, Viner RM, Haller DM, Bose K et al (2009) Global patterns of mortality in young people: a systematic analysis of population health data. Lancet 374(9693):881-892

22. Patel V, Flisher AJ, Hetrick S, Hetrick S, McGorry P (2007) Mental health of young people: a global public-health challenge. Lancet 369(9569):1302-1313

23. Rehm J, Baliunas D, Borges GL, Graham K, Irving HM, Kehoe T et al (2010) The relation between different dimensions of alcohol consumption and burden of disease - an overview. Addiction 105(5):817-843

24. Almeida C, Braveman P, Gold MR, Szwarcwald CL, Ribeiro JM, Miglionico A et al (2001) Methodological concerns and recommendations on policy consequences of the World Health Report 2000. Lancet 357(9269):1692-1697 